\title{
Degree Distribution of Competition-Induced Preferential Attachment Graphs
}

\author{
N. Berger* \\ C. Borgs* \\ J. T. Chayes* \\ R. M. D'Souza* \\ R. D. Kleinberg ${ }^{\dagger}$
}

November 29, 2004

Dedicated to B. Bollobás on the occasion of his 60th birthday.

\begin{abstract}
We introduce a family of one-dimensional geometric growth models, constructed iteratively by locally optimizing the tradeoffs between two competing metrics, and show that this family is equivalent to a family of preferential attachment random graph models with upper cutoffs. This is the first explanation of how preferential attachment can arise from a more basic underlying mechanism of local competition. We rigorously determine the degree distribution for the family of random graph models, showing that it obeys a power law up to a finite threshold and decays exponentially above this threshold.

We also rigorously analyze a generalized version of our graph process, with two natural parameters, one corresponding to the cutoff and the other a "fertility" parameter. We prove that the general model has a power-law degree distribution up to a cutoff, and establish monotonicity of the power as a function of the two parameters. Limiting cases of the general model include the standard preferential attachment model without cutoff and the uniform attachment model.
\end{abstract}

\footnotetext{
*Microsoft Research, One Microsoft Way, Redmond WA 98052, USA

$\dagger$ M.I.T. CSAIL, 77 Massachusetts Ave, Cambridge MA 02139, USA. Supported by a Fannie and John Hertz Foundation Fellowship.
} 


\section{Introduction}

\section{$1.1 \quad$ Network growth models}

This paper is dedicated, with great affection and admiration, to Béla Bollobás on the occasion of his 60 th birthday. Two of us (C.B. and J.T.C.) are privileged to count Béla among our dearest friends. And all of us have been inspired by his pioneering work on graph processes in general, and scale-free graphs in particular. We use the opportunity of this birthday volume to provide complete proofs of results on a new graph model, first announced in [6].

There is currently tremendous interest in understanding the mathematical structure of networks - especially as we discover the pervasiveness of network structures in natural and engineered systems. Much recent theoretical work has been motivated by measurements of real-world networks, indicating they have certain "scale-free" properties, such as a power-law distribution of degrees. For the Internet graph, in particular, both the graph of routers and the graph of autonomous systems (AS) seem to obey power laws [15, 16. However, these observed power laws hold only for a limited range of degrees, presumably due to physical constraints and the finite size of the Internet.

Many random network growth models have been proposed which give rise to power-law degree distributions. Most of these models rely on a small number of basic mechanisms, mainly preferential attachment ${ }^{1}$ [20, 4] or copying [18, extending ideas known for many years [13, 21, 23, 22] to a network context. Variants of the basic preferential attachment mechanism have also been proposed, and some of these lead to changes in the values of the exponents in the resulting power laws. For extensive reviews of work in this area, see Albert and Barabási [2, Dorogovtsev and Mendes [12], and Newman [19] for a survey of the relatively limited amount of mathematical work see $[8$. Most of this work concerns network models without reference to an underlying geometric space. Nor do most of these models allow for heterogeneity of nodes, or address physical constraints on the capacity of the nodes. Thus, while such models may be quite appropriate for geometry-free networks, such as the web graph, they do not seem to be ideally suited to the description of other observed networks, e.g., the Internet graph.

In this paper, instead of assuming preferential attachment, we show that it can arise from a more basic underlying process, namely competition between opposing forces. The idea that power laws can arise from competing effects, modeled as the solution of optimization problems with complex objectives, was proposed originally by Carlson and Doyle [10]. Their "highly optimized tolerance" (HOT) framework has reliable design as a primary objective. Fabrikant, Koutsoupias and Papadimitriou (FKP) [14] introduce an elegant network growth model with such a mechanism, which they called "heuristically optimized trade-offs". As in many growth models, the FKP network is grown one node at a time, with each new node choosing a previous node to which it connects. However, in contrast to the standard preferential attachment types of models, a key feature of the FKP model is the underlying geometry. The nodes are points chosen uniformly at random from some region, for example a unit square in the plane. The trade-off is between the geometric consideration that it is desirable to connect to a nearby point, and a networking consideration, that it is

\footnotetext{
${ }^{1}$ As Aldous 3 points out, proportional attachment may be a more appropriate name, stressing the linear dependence of the attractiveness on the degree.
} 
desirable to connect to a node that is "central" in the network as a graph. Centrality is measured by using, for example, the graph distance to the initial node. The model has a tunable, but fixed, parameter, which determines the relative weights given to the geometric distance and the graph distance.

The suggestion that competition between two metrics could be an alternative to preferential attachment for generating power-law degree distributions represents an important paradigm shift. Though FKP introduced this paradigm for network growth, and FKP networks have many interesting properties, the resulting distribution is not a power law in the standard sense [5]. Instead the overwhelming majority of the nodes are leaves (degree one), and a second substantial fraction heavily connected "stars" (hubs), producing a node degree distribution which has clear bimodal features. ${ }^{2}$

Here, instead of directly producing power laws as a consequence of competition between metrics, we show that such competition can give rise to a preferential attachment mechanism, which in turn gives rise to power laws. Moreover, the power laws we generate have an upper cutoff, which is more realistic in the context of many applications.

\subsection{Overview of competition-induced preferential attachment}

We begin by formulating a general competition model for network growth. Let $x_{0}, x_{1}, \ldots, x_{t}$ be a sequence of random variables with values in some space $\Lambda$. We think of the points $x_{0}, x_{1}, \ldots, x_{t}$ arriving one at a time according to some stochastic process. For example, we typically take $\Lambda$ to be a compact subset of $\mathbb{R}^{d}$, $x_{0}$ to be a given point, say the origin, and $x_{1}, \ldots, x_{t}$ to be i.i.d. uniform on $\Lambda$. The network at time $t$ will be represented by a graph, $G(t)$, on $t+1$ vertices, labeled $0,1, \ldots, t$, and at each time step, the new node attaches to one or several nodes in the existing network. For simplicity, here we assume that each new node connects to a single node, resulting in $G(t)$ being a tree.

Given $G(t-1)$, the new node, labeled $t$, attaches to that node $j$ in the existing network that minimizes a certain cost function representing the trade-off of two competing effects, namely connection or startup cost, and routing or performance cost. The connection cost is represented by a metric, $g_{i j}(t)$, on $\{0, \ldots, t\}$ which depends on $x_{0}, \ldots, x_{t}$, but not on the current graph $G(t-1)$, while the routing cost is represented by a function, $h_{j}(t-1)$, on the nodes which depends on the current graph, but not on the physical locations $x_{0}, \ldots, x_{t}$ of the nodes $0, \ldots, t$. This leads to the cost function

$$
c_{t}=\min _{j}\left[\alpha g_{t j}(t)+h_{j}(t-1)\right]
$$

where $\alpha$ is a constant which determines the relative weighting between connection and routing costs. We think of the function $h_{j}(t-1)$ as measuring the centrality of the node $j$; for simplicity, we take it to be the hop distance along the graph $G(t-1)$ from $j$ to the root 0 .

To simplify the analysis of the random graph process, we will assume that nodes always choose to connect to a point which is closer to the root, i.e., they minimize the cost function

$$
\tilde{c}_{t}=\min _{j:\left\|x_{j}\right\|<\left\|x_{t}\right\|}\left[\alpha g_{t j}(t)+h_{j}(t-1)\right],
$$

\footnotetext{
${ }^{2}$ In simulations of the FKP model, this can be clearly discerned by examining the probability distribution function (pdf); for the system sizes amenable to simulations, it is less prominent in the cumulative distribution function (cdf).
} 
where $\|\cdot\|$ is an appropriate norm.

In the original FKP model, $\Lambda$ is a compact subset of $\mathbb{R}^{2}$, say the unit square, and the points $x_{i}$ are independently uniformly distributed on $\Lambda$. The cost function is of the form (1), with $g_{i j}=d_{i j}$, the Euclidean metric (modeling the cost of building the physical transmission line), and $h_{j}(t)$ is the hop distance along the existing network $G(t)$ from $j$ to the root. A rigorous analysis of the degree distribution of this twodimensional model was given in [5], and the analogous one-dimensional problem was treated in [17.

Our model is defined as follows.

Definition 1 (Border Toll Optimization Process). Let $x_{0}=0$, and let $x_{1}, x_{2}, \ldots$ be i.i.d., uniformly at random in the unit interval $\Lambda=[0,1]$, and let $G(t)$ be the following process: At $t=0, G(t)$ consists of a single vertex 0 , the root. Let $h_{j}(t)$ be the hop distance to 0 along $G(t)$, and let $g_{i j}(t)=n_{i j}(t)$ be the number of existing nodes between $x_{i}$ and $x_{j}$ at time $t$, which we refer to as the jump cost of $i$ connecting to $j$. Given $G(t-1)$ at time $t-1$, a new vertex, labeled $t$, attaches to the node $j$ which minimizes the cost function (2). Furthermore, if there are several nodes $j$ that minimize this cost function and satisfy the constraint, we choose the one whose position $x_{j}$ is nearest to $x_{t}$. The process so defined is called the border toll optimization process (BTOP).

As in the FKP model, the routing cost is just the hop distance to the root along the existing network. However, in our model the connection cost metric measures the number of "borders" between two nodes: hence the name BTOP. Note the correspondence to the Internet, where the principal connection cost is related to the number of AS domains crossed - representing, e.g., the overhead associated with BGP, monetary costs of peering agreements, etc. In order to facilitate a rigorous analysis of our model, we took the simpler cost function (2), so that the new node always attaches to a node to its left.

It is interesting to note that the ratio of the BTOP connection cost metric to that of the one-dimensional FKP model is just the local density of nodes: $n_{i j} / d_{i j}=\rho_{i j}$. Thus the transformation between the two models is equivalent to replacing the constant parameter $\alpha$ in the FKP model with a variable parameter $\alpha_{i j}=\alpha \rho_{i j}$ which changes as the network evolves in time. That $\alpha_{i j}$ is proportional to the local density of nodes in the network reflects a model with an increase in cost for local resources that are scarce or in high demand. Alternatively, it can be thought of as reflecting the economic advantages of being first to market.

Somewhat surprisingly, the BTOP is equivalent to a special case of the following process, which closely parallels the preferential attachment model and makes no reference to any underlying geometry.

Definition 2 (Generalized Preferential Attachment with Fertility and Aging). Let $A_{1}, A_{2}$ be two positive integer-valued parameters. Let $G(t)$ be the following Markov process, whose states are finite rooted trees in which each node is labeled either fertile or infertile. At time $t=0, G(t)$ consists of a single fertile vertex. Given the graph at time $t$, the new graph is formed in two steps: first, a new vertex, labeled $t+1$ and initialized as infertile, connects to an old vertex $j$ with probability zero if $j$ is infertile, and with probability

$$
\operatorname{Pr}(t+1 \rightarrow j)=\frac{\min \left\{d_{j}(t), A_{2}\right\}}{W(t)}
$$

if $j$ is fertile. Here, $d_{j}(t)$ is equal to 1 plus the out-degree of $j$, and $W(t)=\sum_{j}^{\prime} \min \left\{d_{j}(t), A_{2}\right\}$ with the sum running over fertile vertices only. We refer to vertex $t+1$ as a child of $j$. If after the first step, $j$ has more 
than $A_{1}-1$ infertile children, one of them, chosen uniformly at random, becomes fertile. The process so defined is called a generalized preferential attachment process with fertility threshold $A_{1}$ and aging threshold $A_{2}$.

The special case $A_{1}=A_{2}$ is called the competition-induced preferential attachment process with parameter $A_{1}$.

The last definition is motivated by the following theorem, to be proved in Section 2 To state the theorem, we define a graph process as a random sequence of graphs $G(0), G(1), G(2), \ldots$ on the vertex sets $\{0\},\{0,1\},\{0,1,2\}, \ldots$, respectively.

Theorem 1. As a graph process, the border toll optimization process has the same distribution as the competition-induced preferential attachment process with parameter $A=\left\lceil\alpha^{-1}\right\rceil$.

Certain other limiting cases of the generalized preferential attachment process are worth noting. If $A_{1}=1$ and $A_{2}=\infty$, we recover the standard model of preferential attachment as considered in [20, 4]. If $A_{1}=1$ and $A_{2}$ is finite, the model is equivalent to the standard model of preferential attachment with a cutoff. On the other hand, if $A_{1}=A_{2}=1$, we get a uniform attachment model.

The degree distribution of our random trees is characterized by the following theorem, which asserts that almost surely (a.s.) the fraction of vertices having degree $k$ converges to a specified limit $q_{k}$, and moreover that this limit obeys a power law for $k<A_{2}$, and decays exponentially above $A_{2}$.

Theorem 2. Let $A_{1}, A_{2}$ be positive integers. Consider the generalized preferential attachment process with fertility parameter $A_{1}$ and aging parameter $A_{2}$. Let $N_{0}(t)$ be the number of infertile vertices at time $t$, and let $N_{k}(t)$ be the number of fertile vertices with $k-1$ children at time $t, k \geq 1$. Then:

1. There are numbers $q_{k} \in[0,1]$ such that, for all $k \geq 0$

$$
\frac{N_{k}(t)}{t+1} \rightarrow q_{k} \quad \text { a.s., as } t \rightarrow \infty .
$$

2. There exists a number $w=w\left(A_{1}, A_{2}\right) \in[0,2]$ such that the $q_{k}$ are determined by the following equations:

$$
\begin{aligned}
& q_{i}=\left(\prod_{k=2}^{i} \frac{k-1}{k+w}\right) q_{1} \quad \text { if } \quad 1 \leq i \leq A_{2}, \\
& q_{i}=\left(\frac{A_{2}}{A_{2}+w}\right)^{i-A_{2}} q_{A_{2}} \quad \text { if } \quad i>A_{2} \\
& 1=\sum_{i=0}^{\infty} q_{i}, \quad \text { and } \quad q_{0}=\sum_{i=1}^{\infty} q_{i} \min \left\{i-1, A_{1}-1\right\} .
\end{aligned}
$$

3. There are positive constants $c_{1}$ and $C_{1}$, independent of $A_{1}$ and $A_{2}$, such that

$$
c_{1} k^{-(w+1)}<q_{k} / q_{1}<C_{1} k^{-(w+1)}
$$

for $1 \leq k \leq A_{2}$. 
4. If $A_{1}=A_{2}$, the parameter $w$ is equal to 1 , and for general $A_{1}$ and $A_{2}$, $w$ decreases with increasing $A_{1}$, and increases with increasing $A_{2}$.

Equation (77) clearly defines a power-law degree distribution with exponent $\gamma=w+1$ for $k \leq A_{2}$. Note that for measurements of the Internet the value of the exponent for the power law is $\gamma \approx 2$. In our border toll optimization model, where $A_{1}=A_{2}$, we recover $\gamma=2$.

The convergence claim of Theorem 2 is proved using a novel method which we believe is one of the main technical contributions of this work. For preferential attachment models which have been analyzed in the past 11, 1, 9, 11], the convergence was established using the Azuma-Hoeffding martingale inequality. To establish the bounded-differences hypothesis required by that inequality, those proofs employed a clever coupling of the random decisions made by the various edges, such that the decisions made by an edge $e$ only influence the decisions of subsequent edges which choose to imitate $e$ 's choices. A consequence of this coupling is that if $e$ made a different decision, it would alter the degrees of only finitely many vertices. This in turn allows the required bounded-differences hypothesis to be established. No such approach is available for our models, because the coupling fails. The random decisions made by an edge $e$ may influence the time at which some node $v$ crosses the fertility or aging threshold, which thereby exerts a subtle influence on the decisions of every future edge, not only those which choose to imitate $e$.

Instead we introduce a new approach based on the second-moment method. The argument establishing the requisite second-moment upper bound is quite subtle; it depends on a computation involving the eigenvalues of a matrix describing the evolution of the degree sequence in a continuous-time version of the model.

\section{Equivalence of the Two Models}

\subsection{Basic properties of the border toll optimization process}

In this section we will turn to the BTOP defined in the introduction, establishing some basic properties which will enable us to prove that it is equivalent to the competition-induced preferential attachment model. In order to avoid complications we exclude the case that some of the $x_{i}$ 's are identical, an event that has probability zero. We say that $j \in\{0,1 \ldots, t\}$ lies to the right of $i \in\{0,1 \ldots, t\}$ if $x_{i}<x_{j}$, and we say that $j$ lies directly to the right of $i$ if $x_{i}<x_{j}$ but there is no $k \in\{1, \ldots, t\}$ such that $x_{i}<x_{k}<x_{j}$. In a similar way, we say that $j$ is the first vertex with a certain property to the right of $i$ if $j$ has that property and there exists no $k \in\{1, \ldots, t\}$ such that $x_{i}<x_{k}<x_{j}$ and $k$ has the property in question. Similar notions apply with "left" in place of "right".

Definition 3. A vertex $i$ is called fertile at time $t$ if a hypothetical new point arriving at time $t+1$ and landing directly to the right of $x_{i}$ would attach itself to the node $i$. Otherwise $i$ is called infertile at time $t$.

This definition is illustrated in Fig. [1]

Lemma 3. Let $0<\alpha<\infty$, let $A=\left\lceil\alpha^{-1}\right\rceil$, and let $0<t<\infty$. Then

i) The node 0 is fertile at time $t$. 


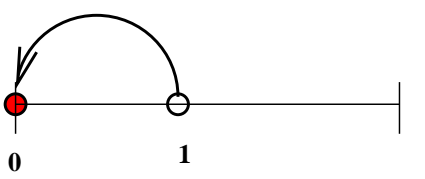

$\mathrm{t}=1$

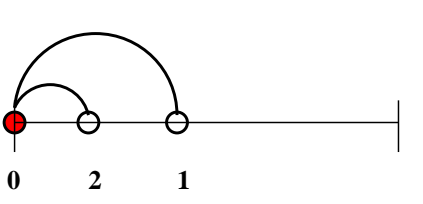

$\mathrm{t}=2$
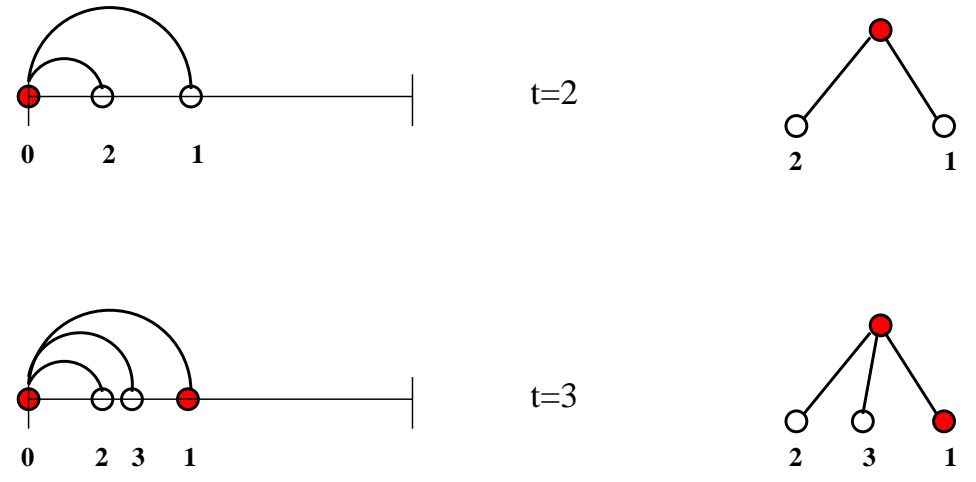

$\mathrm{t}=3$
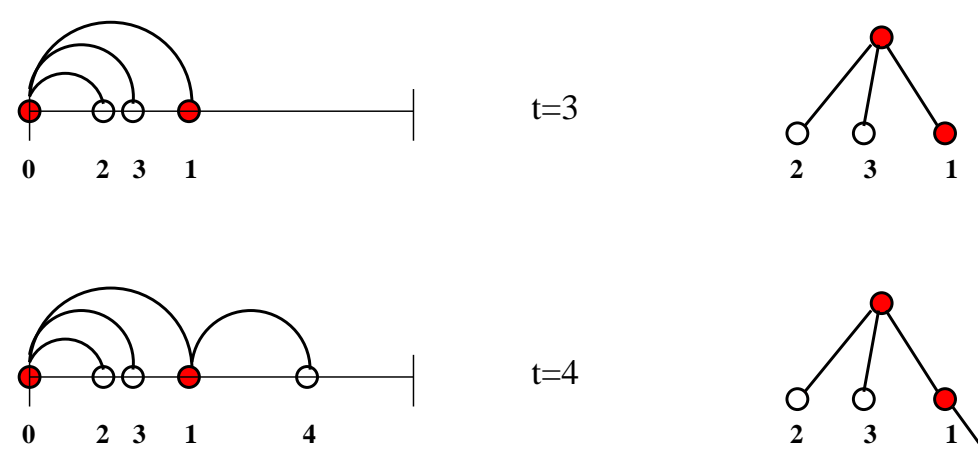

$\mathrm{t}=4$

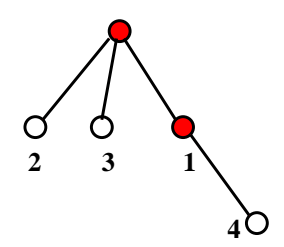

Figure 1: A sample instance of BTOP for $\alpha=1 / 3, A=3$, showing the process on the unit interval (on the left), and the resulting tree (on the right). Fertile vertices are shaded, infertile ones are not. Note that vertex 1 became fertile at $t=3$.

ii) Let $i$ be fertile at time $t$. If $i$ is the rightmost fertile vertex at time $t$ (case 1), let $\ell$ be the number of infertile vertices to the right of $i$. Otherwise (case 2), let $j$ be the next fertile vertex to the right of $i$, and let $\ell=n_{i j}(t)$. Then $0 \leq \ell \leq A-1$, and the $\ell$ infertile vertices located directly to the right of $i$ are children of $i$. In case 2 , if $h_{j}>h_{i}$, then $j$ is a fertile child of $i$ and $\ell=A-1$. As a consequence, the hop count between two consecutive fertile vertices never increases by more than 1 as we move to the right, and if it increases by 1 , there are $A-1$ infertile vertices between the two fertile ones.

iii) Assume that the new vertex at time $t+1$ lands between two consecutive fertile vertices $i$ and $j$, and let $\ell=n_{i j}(t)$. Then $t+1$ becomes a child of $i$. If $\ell+1<A$, the new vertex is infertile at time $t+1$, and the fertility of all old vertices is unchanged. If $\ell+1=A$ and the new vertex lies directly to the left of $j$, the new vertex is fertile at time $t+1$ and the fertility of the old vertices is unchanged. If $\ell+1=A$ and the new vertex does not lie directly to the left of $j$, the new vertex is infertile at time $t+1$, the vertex directly to the left of $j$ becomes fertile, and the fertility of all other vertices is unchanged.

iv) If $t+1$ lands to the right of the rightmost fertile vertex at time $t$, the statements in iii) hold with $j$ replaced by the right endpoint of the interval $[0,1]$, and $n_{i j}(t)$ replaced by the number of vertices to the right of $i$.

v) If $i$ is fertile at time $t$, it is still fertile at time $t+1$. 
vi) If $i$ has $k$ children at time $t$, the $\ell=\min \{A-1, k\}$ leftmost of them are infertile at time $t$, and any others are fertile.

Proof. The proof is straightforward but lengthy. We include the details of the argument here for completeness.

Statement i) is trivial, statement v) follows immediately from iii) and iv), and vi) follows immediately from ii). So we are left with ii) - iv). We proceed by induction on $t$. If ii) holds at time $t$, and iii) and iv) hold for a new vertex arriving at time $t+1$, ii) clearly also holds at time $t+1$. We therefore only have to prove that ii) at time $t$ implies iii) and iv) for a new vertex arriving at time $t+1$.

Assume thus that ii) holds at time $t$. At time $t+1$, a new vertex arrives, and falls directly to the right of some vertex $k$. Let $i$ be the nearest vertex to the left of $k$ that was fertile at time $t$ (if $k$ is fertile at time $t$, we set $i=k$ ) and let $j$ be the nearest vertex to the right of $i$ that was fertile at time $t$ (we assume for the moment that $i$ is not the rightmost fertile vertex at time $t$ ), let $\ell$ be the number of vertices between $i$ and $j$ at time $t$.

Let us first prove that the vertex $t+1$ connects to $i$. If $i=k$, this is obvious, since $i$ is fertile at time $t$. We may therefore assume that $k \neq i$. For the new vertex $t+1$, the cost of connecting to the vertex $i$ is then equal to $\alpha\left(n_{i k}(t)+1\right)$. Let us first compare this cost to the cost of connecting to a fertile vertex $i^{\prime}$ to the left of $i$. Let $i_{0}=i^{\prime}$, let $i_{s}=i$, and let $i_{1}, \ldots, i_{s-1}$ be the fertile vertices between $i^{\prime}$ and $i$, ordered from left to right. If $h_{i_{m-1}}<h_{i_{m}}$, we use the inductive assumption ii) to conclude that the number of infertile vertices between $i_{m-1}$ and $i_{m}$ is equal to $A-1$, and $h_{i_{m-1}}=h_{i_{m}}-1$. A decrease of $q$ in the hop cost is therefore accompanied by an increase in the jump cost of at least $\alpha A q \geq q$. As a consequence, it never pays to connect to a fertile vertex $i^{\prime}$ to the left of $i$. The cost of connecting to an infertile vertex to the left of $i$ is even higher, since the hop count of an infertile vertex is at best equal to the hop count of the next fertile vertex to the right. We therefore only have to consider the connection cost to some of the infertile children of $i$. But again, the hop count is worse by 1 when compared to the hop count of $i$, and the jump cost is at best reduced by $(A-1) \alpha<1$, proving that the cost of connecting to $i$ is minimal.

To discuss the fertility of the vertices in the graph $G(t+1)$, we need to consider the arrival of a second vertex, labeled $t+2$. If $t+2$ falls to the left of $t+1$, it will face an optimization problem that has not been changed by the arrival of the vertex $t+1$, implying that the fertility of the vertices to the left of $t+1$ is unchanged. If $t+2$ falls to the right of $j$, the cost of connecting to $j$ or one of the vertices to the right of $j$ is the same as before, and the cost of connecting to a vertex to the left of $j$ is at best equal (the cost of connecting to any vertex to the left of $t+1$ is in fact higher, due to the additional cost of jumping over the vertex $t+1)$. Therefore, the vertex $t+2$ will still prefer to connect to either $j$ or one of the vertices to the right of $j$, implying that the fertility of the vertices to the right of $j$ has not changed at all. We therefore are left with analyzing the case where $t+2$ falls between $t+1$ and $j$. Again, the vertex $t+2$ will prefer $i$ over any vertex to the left of $i$ (the cost analysis is the same as the one used for $t+1$ above), so we just have to compare the costs of connecting to the different vertices between $i$ and $j$. If $\ell+1<A$, this will again imply that $t+2$ connect to $i$; but if $\ell+1=A$, the vertex $t+2$ will only connect to $i$ if it does not fall to the right of the rightmost of the now $\ell+1$ vertices between $i$ and $j$. If it falls to the right of this vertex, it will be as expensive to connect to the rightmost of the now $\ell+1$ vertices between $i$ and $j$ as it is to connect to $i$. 
Recalling out convention of connecting to the nearest vertex to the left if there is a tie in costs, this proves that now $t+2$ connects to the rightmost vertex between $i$ and $j$, implying that this vertex is fertile.

The above considerations prove the fertility statements in iii), and thus completes the proof of iii). The case where $i$ is the rightmost fertile vertex at time $t$ is similar (in fact, it is slightly easier since it involves fewer cases), and leads to the proof of iv). This completes the proof of Lemma 3 .

\subsection{Proof of Theorem 1}

In the BTOP, note that our cost function

$$
\min _{\mathrm{j}}\left[\alpha n_{t j}(t)+h_{j}(t-1)\right]
$$

and hence the graph $G(t)$, only depends on the order of the vertices $x_{0}, \ldots, x_{t}$, and not on their actual positions in the interval $[0,1]$. Let $\vec{\pi}(t)$ be the permutation of $\{0,1, \ldots, t\}$ which orders the vertices $x_{0}, \ldots, x_{t}$ from left to right, so that

$$
x_{0}=x_{\pi_{0}(t)}<x_{\pi_{1}(t)}<\cdots<x_{\pi_{t}(t)}
$$

(Recall that the vertices $x_{0}, x_{1}, \ldots, x_{t}$ are pairwise distinct with probability one.) Note that $\vec{\pi}(t)$ and $\vec{\pi}(t+1)$ are related as follows: there exists $i_{0} \in\{1,2, \ldots, t+1\}$ such that

$$
\pi_{i}(t+1)=\left\{\begin{array}{lll}
\pi_{i}(t) & \text { if } & i<i_{0} \\
t+1 & \text { if } \quad i=i_{0} \\
\pi_{i-1}(t) & \text { if } \quad i>i_{0} .
\end{array}\right.
$$

Informally, the permutation $\vec{\pi}(t+1)$ is obtained by inserting the new element $t+1$ into the permutation $\vec{\pi}(t)$ in a random position $i_{0}$, where $x_{\pi_{i_{0}}(t)}$ is the left endpoint of the subinterval of $(0,1)$ into which $x_{t+1}$ falls. The distribution of the random variable $i_{0}$ may be deduced as follows. Since $x_{0}=0$ and $x_{1}, x_{2}, \ldots, x_{t}$ are i.i.d., we know that, for all $t$, the permutation $\vec{\pi}(t)$ is uniformly distributed among permutations of $\{0,1, \ldots, t\}$ which fix the element 0 . This means that, conditioned on a given such permutation $\vec{\pi}(t)$, the permutation $\vec{\pi}(t+1)$ is uniformly distributed among all permutations related to $\vec{\pi}(t)$ by the transformation (10). In other words, $i_{0}$ is uniformly distributed in the set $\{1,2, \ldots, t+1\}$.

With the help of Lemma 3] we now easily derive a description of the graph $G(t)$ which does not involve any optimization problem. To this end, let us consider a vertex $i$ with $\ell$ infertile children at time $t$. If a new vertex falls into the interval directly to the right of $i$, or into one of the intervals directly to the right of an infertile child of $i$, it will connect to the vertex $i$. Since there is a total of $t+1$ intervals at time $t$, the probability that a vertex $i$ with $\ell$ infertile children grows an offspring is $(\ell+1) /(t+1)$. By Lemma 3 (vi), this number is equal to $\min \left\{A, k_{i}\right\} /(t+1)$, where $k_{i}-1$ is the number of children of $i$. Note that fertile children do not contribute to this probability, since vertices falling into an interval directly to the right of a fertile child will connect to the child, not the parent.

Assume now that $i$ did get a new offspring, and that it had $A-1$ infertile children at time $t$. Then the new vertex is either born fertile, or makes one of its infertile siblings fertile. Using the principle of deferred 
decisions, we may assume that with probability $1 / A$ the new vertex becomes fertile, and with probability $(A-1) / A$ an old one, chosen uniformly at random among the $A-1$ candidates, becomes fertile.

We thus have shown that the solution $G(t)$ of the optimization problem (8) can alternatively be described by the competition-induced preferential attachment model with parameter $A$.

\section{Convergence of the Degree Distribution}

\subsection{Overview}

To characterize the behavior of the degree distribution, we will derive a recursion which governs the evolution of the vector $\vec{N}(t)$, whose components are the number of vertices of each degree, at the time when there are $t$ nodes in the network. The conditional expectation of $\vec{N}(t+1)$ is given by an evolution equation of the form

$$
\mathbb{E}(\vec{N}(t+1)-\vec{N}(t) \mid \vec{N}(t))=M(t) \vec{N}(t),
$$

where $M(t)$ depends on $t$ through the random variable $W(t)$ introduced in Definition 2 Due to the randomness of the coefficient matrix $M(t)$, the analysis of this evolution equation is not straightforward. We avoid this problem by introducing a continuous-time process, with time parameter $\tau$, which is equivalent to the original discrete-time process up to a (random) reparametrization of the time coordinate. The evolution equation for the conditional expectations in the continuous-time process involves a coefficient matrix $M$ that is not random and does not depend on $\tau$. We will first prove that the expected degree distribution in the continuous-time model converges to a scalar multiple of the eigenvector $\hat{p}$ of $M$ associated with the largest eigenvalue $w$. This is followed by the much more difficult proof that the empirical degree distribution converges a.s. to the same limit. Finally, we translate this continuous-time result into a rigorous convergence result for the original discrete-time system.

\subsection{Notation}

Let $A$ be any integer greater than or equal to $\max \left(A_{1}, A_{2}\right)$. Let $N_{0}(t)$ be the number of infertile vertices at (discrete) time $t$, and, for $k \geq 1$, let $N_{k}(t)$ be the number of fertile vertices with $k-1$ children at time $t$. Let $\widetilde{N}_{A}(t)=N_{\geq A}(t)=\sum_{k \geq A} N_{k}(t)$, and $\widetilde{N}_{k}(t)=N_{k}(t)$ if $k<A$. The combined attractiveness of all vertices is denoted by $W(t)=\sum_{k=1}^{A} \min \left\{k, A_{2}\right\} \widetilde{N}_{k}(t)$. Let $n_{k}(t)=\frac{1}{t+1} N_{k}(t)$ and $\tilde{n}_{k}(t)=\frac{1}{t+1} \widetilde{N}_{k}(t)$. Finally, the vectors $\left(\tilde{N}_{k}(t)\right)_{k=1}^{A}$ and $\left(\tilde{n}_{k}(t)\right)_{k=1}^{A}$ are denoted by $\tilde{N}(t)$ and $\tilde{n}(t)$ respectively. Note that the index $k$ runs from 1 to $A$, not 0 to $A$.

\subsection{Evolution of the expected value}

From the definition of the generalized preferential attachment model, it is easy to derive the probabilities for the various alternatives which may happen upon the arrival of the $(t+1)$-st node:

- With probability $A_{2} \widetilde{N}_{A}(t) / W(t)$, it attaches to a node of degree $\geq A$. This increments $\widetilde{N}_{1}$, and leaves $\widetilde{N}_{A}$ and all $\widetilde{N}_{j}$ with $1<j<A$ unchanged. 
- With probability $\min \left(A_{2}, k\right) \widetilde{N}_{k}(t) / W(t)$, it attaches to a node of degree $k$, where $1 \leq k<A$. This increments $\widetilde{N}_{k+1}$, decrements $\widetilde{N}_{k}$, increments $\widetilde{N}_{0}$ or $\widetilde{N}_{1}$ depending on whether $k<A_{1}$ or $k \geq A_{1}$, and leaves all other $\widetilde{N}_{j}$ with $j<A$ unchanged.

It follows that the discrete-time process $\left(\widetilde{N}_{k}(t)\right)_{k=0}^{A}$ at time $t$ is equivalent to the state of the following continuous-time stochastic process $\left(\widehat{N}_{k}(\tau)\right)_{k=0}^{A}$ at the random stopping time $\tau=\tau_{t}$ of the $t$-th event.

- With rate $A_{2} \widehat{N}_{A}(\tau), \widehat{N}_{1}$ increases by 1 .

- For every $0<k<A$, with rate $\widehat{N}_{k}(\tau) \min \left(k, A_{2}\right)$, the following happens:

$$
\widehat{N}_{k} \rightarrow \widehat{N}_{k}-1 \quad ; \quad \widehat{N}_{k+1} \rightarrow \widehat{N}_{k+1}+1 \quad ; \quad \widehat{N}_{g(k)} \rightarrow \widehat{N}_{g(k)}+1
$$

where $g(k)=0$ for $k<A_{1}$ and $g(k)=1$ otherwise.

Note that the above rules need to be modified if $A_{1}=1$. Here the birth of a child of a degree-one vertex does not change the net number of fertile degree-one vertices, $N_{1}$.

Let $M$ be the following $A \times A$ matrix:

$$
M_{i, j}= \begin{cases}-1 & \text { if } i=j=1<A_{1} \\ -\min \left(j, A_{2}\right) & \text { if } 2 \leq i=j \leq A-1 \\ \min \left(j, A_{2}\right) & \text { if } 2 \leq i=j+1 \leq A \\ \min \left(j, A_{2}\right) & \text { if } i=1 \text { and } j \geq \max \left(A_{1}, 2\right) \\ 0 & \text { otherwise. }\end{cases}
$$

Then, for every $\tau>\sigma$, the conditional expectation of the vector $\widehat{N}(\tau)=\left(\widehat{N}_{k}(\tau)\right)_{k=1}^{A}$ is given by

$$
\mathbb{E}(\widehat{N}(\tau) \mid \widehat{N}(\sigma))=e^{(\tau-\sigma) M} \widehat{N}(\sigma) .
$$

It is easy to see that the matrix $e^{M}$ has all positive entries, and therefore (by the Perron-Frobenius Theorem) $M$ has a unique eigenvector $\hat{p}$ of $\ell_{1}$-norm 1 having all positive entries. Let $w$ be the eigenvalue corresponding to $\hat{p}$. Then $w$ is real, it has multiplicity 1 , and it exceeds the real part of every other eigenvalue. Therefore, for every non-zero vector $y$ with non-negative entries,

$$
\lim _{\tau \rightarrow \infty} e^{-\tau w} e^{\tau M} y=\langle\hat{a}, y\rangle \hat{p}
$$

where $\hat{a}$ is the eigenvector of $M^{\top}$ corresponding to $w$, normalized so that $\langle\hat{a}, \hat{p}\rangle=1$. Note that $\langle\hat{a}, y\rangle>0$ because $y$ is non-zero and non-negative, and $\hat{a}$ is positive, again by Perron-Frobenius. Therefore, the vector $\mathbb{E}\left(e^{-\tau w} \widehat{N}(\tau)\right)$ converges to a positive scalar multiple of $\hat{p}$, say $\lambda \hat{p}$, as $\tau \rightarrow \infty$.

In order to prove concentration for the continuous-time model, we will prove that the difference $\widehat{N}_{k}(\tau) / q_{k}-$ $\widehat{N}_{j}(\tau) / q_{j}$ has an exponential growth rate which is at most the real part of the second eigenvalue of $M$, which is strictly less than $w$, the growth rate of the individual terms $\widehat{N}_{k}(\tau) / q_{k}$ and $\widehat{N}_{j}(\tau) / q_{j}$. From this, we will conclude that the ratio $\widehat{N}_{k}(\tau) / \widehat{N}_{j}(\tau)$ converges almost surely to $q_{k} / q_{j}$, for all $k$ and $j$, which in turn implies the convergence of the normalized degree sequence to the vector $\left(q_{i}\right)_{i=0}^{\infty}$.

In order to prove bounds on the growth rate of the differences $\widehat{N}_{k}(\tau) / q_{k}-\widehat{N}_{j}(\tau) / q_{j}$, we will need some auxiliary bounds involving the well-known standard birth process, to be defined below. 


\subsection{Standard birth process}

We start with the definition of the standard birth process with rate $\rho$. The standard birth process was first introduced by Yule in 1924 [22], and is a special case of the well known Yule Process, defined in that paper.

Definition 4. Let $\rho>0$ and let $\left\{o_{n}\right\}_{n=1}^{\infty}$ be independent exponential random variables so that $\mathbb{E}\left(o_{n}\right)=\frac{1}{\rho} n^{-1}$. For $\tau \in[0, \infty)$, let $X_{\tau}=\min \left\{n \geq 1: \sum_{k=1}^{n} o_{k}>\tau\right\}$. Then $X$ is called the standard birth process with rate $\rho .^{3}$

The standard birth process is connected to our discussion through the following easy claim:

Claim 1. Let $\|\widehat{N}(\tau)\|=\sum_{k=1}^{A} \widehat{N}_{k}(\tau)$. Let $T \geq 0$, let $x \geq y$, and let $X$ be a standard birth process with rate 2. Then $\left\{\left\{X_{\tau}\right\}_{\tau \geq T} \mid X_{T}=x\right\}$ stochastically dominates $\left\{\{\|\widehat{N}(\tau)\|\}_{\tau \geq T} \mid\|\widehat{N}(T)\|=y\right\}$.

Proof. Let us start with the observation that $\sum_{k=1}^{n} o_{k}$ is the first time $\tau$ for which $X_{\tau}=n+1$. Let $\left\{r_{n}\right\}_{n=0}^{\infty}$ be i.i.d. exponential random variables with mean 1. Then $\sum_{k=1}^{n} o_{k}$ has the same distribution as $\sum_{k=0}^{n-1} r_{k} /(2 k+2)$. The time $\tau_{n}$ at which the node $n$ is born has the same distribution as $\sum_{k=0}^{n-1} r_{k} / W(k)$, where $W(k)$ denotes the combined attractiveness of all nodes at the random time $\tau_{k}$. The claim follows now from the observation that $W(k) \leq 2 k+1 \leq 2 k+2$.

The main purpose of this section is the proof of the following claims.

Claim 2. Let $X$ be a standard birth process with rate $\rho$. Then $X_{\tau}$ is almost surely finite for every $\tau$. Furthermore, there exists a constant $C_{s}=C_{s}(\rho)$ such that for every $\tau_{2}>\tau_{1}, x \geq 1$, and $k \geq 1$,

$$
\mathbf{P}\left(X_{\tau_{2}}>k x e^{\rho\left(\tau_{2}-\tau_{1}\right)} \mid X_{\tau_{1}}=x\right)<\frac{C_{s}}{x(k-1)^{2}} .
$$

If, in addition, $\tau_{2}-\tau_{1}<1$, then

$$
\mathbf{P}\left(X_{\tau_{2}}-X_{\tau_{1}}>k x\left[e^{\rho\left(\tau_{2}-\tau_{1}\right)}-1\right] \mid X_{\tau_{1}}=x\right)<\frac{C_{s}}{x\left(\tau_{2}-\tau_{1}\right)(k-1)^{2}} .
$$

To see the finiteness of $X_{\tau}$, we need to show that $\sum_{n=1}^{\infty} o_{n}=\infty$ a.s. This follows from the following simple argument: For every $k$, Let

$$
U_{k}=\sum_{j=2^{k}+1}^{2^{k+1}} o_{j} .
$$

For $j \in\left[2^{k}+1,2^{k+1}\right]$, with probability greater than $\frac{1}{2}, o_{j}>\frac{1}{\rho} 2^{-k-2}$. Therefore, $\mathbf{P}\left(U_{k}>\frac{1}{4 \rho}\right)>\frac{1}{2}$. The random variables $\left\{U_{k}\right\}_{k=1}^{\infty}$ are independent, and therefore $\sum_{n=1}^{\infty} o_{n} \geq \sum_{k=1}^{\infty} U_{k}=\infty$ almost surely.

To see (13) and (14), we use the following Lemma, which is proved in section II of [22. Since the proof is short and simple, we choose to include it for the sake of making the exposition more self-contained.

Lemma 4 (Yule, 1924). For every $\tau>0$ and every positive integer $k, \mathbb{E}\left(X_{\tau}^{k}\right)<\infty$. Furthermore,

$$
\mathbb{E}\left(X_{\tau}\right)=\exp (\rho \tau)
$$

\footnotetext{
${ }^{3}$ The name "standard birth process" is due to the fact that $X_{\tau}$ is equivalent to the following process: Start with one cell at time 0. At each time, every cell divides into two cells with rate $\rho$. Then $X_{\tau}$ is the number of cells at time $\tau$.
} 
and

$$
\operatorname{var}\left(X_{\tau}\right)=\exp (2 \rho \tau)-\exp (\rho \tau)
$$

In particular,

$$
\operatorname{var}\left(X_{\tau}\right)=O(\exp (2 \rho \tau)),
$$

and for $\tau<1$ there exists a constant $C_{v}=C_{v}(\rho)$ so that

$$
\operatorname{var}\left(X_{\tau}\right) \leq C_{v} \tau \text {. }
$$

Proof. An equivalent description of the standard birth process is the following: Let $\alpha$ be an exponential variable with expected value $\rho$, and let $\left\{G_{t}\right\}$ be a Poisson point process with rate $\alpha e^{\rho t}$. Then $X_{\tau}=1+G_{\tau}$ has the same distribution as the standard birth process. To see this, all we need is to show that for every $\tau$ and $n$, the rate of the process $\left\{G_{t}\right\}$ at time $\tau$ conditioned on $X_{\tau}=n$ is $\rho n$. Indeed,

$$
\begin{aligned}
\operatorname{rate}\left(\tau \mid X_{\tau}=n\right) & =\frac{\int_{0}^{\infty} \alpha e^{\rho \tau} \mathbf{P}\left(X_{\tau}=n \mid \alpha\right) \frac{1}{\rho} e^{-\alpha / \rho} d \alpha}{\int_{0}^{\infty} \mathbf{P}\left(X_{\tau}=n \mid \alpha\right) \frac{1}{\rho} e^{-\alpha / \rho} d \alpha} \\
& =\frac{\int_{0}^{\infty} \alpha e^{\rho \tau} e^{-\frac{\alpha}{\rho}(\exp (\rho \tau)-1)}\left(\frac{\alpha}{\rho}(\exp (\rho \tau)-1)\right)^{(n-1)}((n-1) !)^{-1} \frac{1}{\rho} e^{-\alpha / \rho} d \alpha}{\int_{0}^{\infty} e^{-\frac{\alpha}{\rho}(\exp (\rho \tau)-1)}\left(\frac{\alpha}{\rho}(\exp (\rho \tau)-1)\right)^{(n-1)}((n-1) !)^{-1} \frac{1}{\rho} e^{-\alpha / \rho} d \alpha} \\
& =\rho n .
\end{aligned}
$$

Here the second equality follows from the fact that $X_{\tau}-1$ is a Poisson variable with rate $\frac{\alpha}{\rho}\left(e^{\rho \tau}-1\right)$, and last equality follows by integration by parts.

From this we get that the distribution of $X_{\tau}$ is geometric with expected value $\exp (\rho \tau)$. To see this, we again use the fact that $X_{\tau}-1$ is a Poisson variable with rate $\frac{\alpha}{\rho}\left(e^{\rho \tau}-1\right)$ where $\alpha$ is an exponential variable with expectation $\rho$. Therefore, for every $n$,

$$
\begin{aligned}
\mathbf{P}\left(X_{\tau}=n+1\right) & =\int_{0}^{\infty} \mathbf{P}\left(X_{\tau}-1=n \mid \alpha\right) \frac{1}{\rho} e^{-\alpha / \rho} d \alpha \\
& =(n !)^{-1} \int_{0}^{\infty} e^{-\frac{\alpha}{\rho}\left(e^{\rho \tau}-1\right)}\left(\frac{\alpha}{\rho}\left(e^{\rho \tau}-1\right)\right)^{n} \frac{1}{\rho} e^{-\alpha / \rho} d \alpha=\left(1-e^{-\rho \tau}\right) \mathbf{P}\left(X_{\tau}=n\right)
\end{aligned}
$$

where, again, the last step follows from integration by parts.

The relations (15) and (16) follow immediately, and (17) and (18) follow from (16).

Proof of (13) and 14) in Claim Q Equations (13) and (14) will follow from Chebyshev's inequality if we show that

$$
\mathbb{E}\left(X_{\tau_{2}} \mid X_{\tau_{1}}\right)=X_{\tau_{1}} e^{\rho\left(\tau_{2}-\tau_{1}\right)}
$$

and

$$
\operatorname{var}\left(X_{\tau_{2}} \mid X_{\tau_{1}}\right)=X_{\tau_{1}} O\left(e^{2 \rho\left(\tau_{2}-\tau_{1}\right)}\right)
$$

for $\tau_{2}>\tau_{1}$, and

$$
\operatorname{var}\left(X_{\tau+\tau_{1}} \mid X_{\tau_{1}}\right)=O(\tau) \cdot X_{\tau_{1}}
$$

for $\tau<1$.

Equations (19), (20) and (21) follow from (respectively) (15), (17) and (18) and the fact that conditioned on $X_{\tau_{1}}$, the process $X_{\tau+\tau_{1}}$ is the sum of $X_{\tau_{1}}$ independent copies of $X_{\tau}$. 
Remark: From now on we will always assume that $\rho=2$. In particular, whenever we use the term "standard birth process", it should be understood as "standard birth process with rate 2 ".

\subsection{Concentration of the continuous-time process}

In order to show concentration of the degree distribution for the continuous-time process, we will prove first the following lemma. To state it, we observe for any $b$ with $b^{\top} \hat{p}=0$,

$$
\left\|b^{\top} e^{(T-\tau) M}\right\|_{\infty} \leq\|b\|_{\infty} e^{(T-\tau) v^{\prime}}
$$

for some $v^{\prime}<w$. Without loss of generality, we may assume that $v^{\prime}>w / 2$. Also, for a general vector $b$,

$$
\left\|b^{\top} e^{(T-\tau) M}\right\|_{\infty} \leq\|b\|_{\infty} e^{(T-\tau) w} .
$$

Lemma 5. Let $b$ be a vector in $\mathbb{R}^{A}$ with $\|b\|_{\infty} \leq 1$. Then there exists a constant $C<\infty$, such that for all $T>0$,

$$
\operatorname{var}\left(b^{\top} \widehat{N}(T)\right)<C \exp (2 u T)
$$

where $u=w$ if $b^{\top} \hat{p} \neq 0$, and $u=v^{\prime}$ if $b^{\top} \hat{p}=0$.

Proof. We use a martingale to bound the variance. Fix $T$, and let

$$
L_{\tau}=\mathbb{E}\left(b^{\top} \widehat{N}(T) \mid \widehat{N}(\tau)\right) .
$$

Clearly, $L_{\tau}$ is a (continuous-time) martingale. By (12), we know that $L_{\tau}=b^{\top} e^{(T-\tau) M} \widehat{N}(\tau)$. Let $0<\epsilon<$ $\exp (-10 T)$ be such that $K=T / \epsilon$ is an integer number. Then, $\left\{U_{k}=L_{k \epsilon}\right\}_{k=0}^{K}$ is a martingale and

$$
\operatorname{var}\left(b^{\top} \widehat{N}(T)\right)=\sum_{k=0}^{K-1} \operatorname{var}\left(U_{k+1}-U_{k}\right) .
$$

We want to estimate the variance of $U_{k+1}-U_{k}$. Let $v_{k}=\widehat{N}((k+1) \epsilon)-\widehat{N}(k \epsilon)$. For two vectors $\widehat{N}_{1}$ and $\widehat{N}_{2}$,

$$
\left(b^{\top} e^{(T-(k+1) \epsilon) M} \widehat{N}_{1}-b^{\top} e^{(T-(k+1) \epsilon) M} \widehat{N}_{2}\right)^{2} \leq\left\|\widehat{N}_{1}-\widehat{N}_{2}\right\|^{2} e^{2 u(T-(k+1) \epsilon)},
$$

where the norm $\|\cdot\|$ refers to the $L^{1}$-norm here and throughout this section, unless otherwise noted. Choose $\widehat{N}(k \epsilon)$ according to its distribution, and let $\widehat{N}_{1}$ and $\widehat{N}_{2}$ be chosen independently, according to the distribution of $\widehat{N}((k+1) \epsilon)$ conditioned on $\widehat{N}(k \epsilon)$. Then

$$
\operatorname{var}\left(U_{k+1}-U_{k}\right)=\frac{1}{2} \mathbb{E}\left[\left(b^{\top} e^{(T-(k+1) \epsilon) M} \widehat{N}_{1}-b^{\top} e^{(T-(k+1) \epsilon) M} \widehat{N}_{2}\right)^{2}\right] \leq \frac{1}{2} \mathbb{E}\left(\left\|\widehat{N}_{1}-\widehat{N}_{2}\right\|^{2}\right) e^{2 u(T-(k+1) \epsilon)} .
$$

On the other hand, using the fact that for every vector $x$ in $\mathbb{R}^{d}$,

$$
\left(\sum_{i=1}^{d} x_{i}\right)^{2} \leq d \sum_{i=1}^{d} x_{i}^{2}
$$

we get

$$
\sum_{j=1}^{A} \operatorname{var}\left(v_{k}(j)\right) \geq \frac{1}{2 A} \mathbb{E}\left(\left\|\widehat{N}_{1}-\widehat{N}_{2}\right\|^{2}\right)
$$


where $v_{k}(j)$ is the $j$-th component of $v_{k}$. Therefore,

$$
\operatorname{var}\left(U_{k+1}-U_{k}\right) \leq A \sum_{j=1}^{A} \exp [2 u(T-(k+1) \epsilon)] \operatorname{var}\left(v_{k}(j)\right) .
$$

By Claim 11 (19), and (21), for every $j=1,2, \ldots, A$,

$$
\begin{gathered}
\operatorname{var}\left(v_{k}(j) \mid \widehat{N}(k \epsilon)\right) \leq \mathbb{E}\left[\left(v_{k}(j)\right)^{2} \mid \widehat{N}(k \epsilon)\right] \leq C_{v} \epsilon\|\widehat{N}(k \epsilon)\|, \\
\left|\mathbb{E}\left(v_{k}(j) \mid \widehat{N}(k \epsilon)\right)\right| \leq\left(e^{2 \epsilon}-1\right)\|\widehat{N}(k \epsilon)\| \leq 4 \epsilon\|\widehat{N}(k \epsilon)\|
\end{gathered}
$$

and

$$
\mathbb{E}\left[(\|\widehat{N}(k \epsilon)\|)^{2}\right]<e^{4 k \epsilon} .
$$

Therefore,

$$
\begin{aligned}
\operatorname{var}\left(v_{k}(j)\right) & =\mathbb{E}\left(\operatorname{var}\left(v_{k}(j) \mid \widehat{N}(k \epsilon)\right)\right)+\operatorname{var}\left(\mathbb{E}\left(v_{k}(j) \mid \widehat{N}(k \epsilon)\right)\right) \\
& \leq C_{v} \epsilon \exp (w k \epsilon)+16 \epsilon^{2} \exp (4 k \epsilon) \\
& <C_{0} \epsilon \exp (w k \epsilon)
\end{aligned}
$$

for $C_{0}=C_{v}+1$, by the choice of $\epsilon$. Therefore,

$$
\begin{aligned}
\operatorname{var}\left(b^{\top} \widehat{N}_{k}(T)\right) & <A^{2} C_{0} \epsilon \sum_{k=0}^{K-1} \exp (w k \epsilon+2 u(T-(k+1) \epsilon)) \\
& \leq A^{2} C_{0} e^{2 u T} \int_{0}^{T} e^{(w-2 u) \tau} d \tau<C_{u} \exp (2 u T)
\end{aligned}
$$

for

$$
C_{u}=A^{2} C_{0} \int_{0}^{\infty} e^{(w-2 u) \tau} d \tau<\infty
$$

In addition, note that by (22) and (23),

$$
\left|\mathbb{E}\left(b^{\top} \widehat{N}(T)\right)\right| \leq e^{u T}
$$

and therefore there exists $C$ so that

$$
\mathbb{E}\left[\left(b^{\top} \widehat{N}(T)\right)^{2}\right] \leq C e^{2 u T} .
$$

We are now ready to state and prove the two main lemmas used to prove concentration:

Lemma 6. For every $w^{\prime}<w$ and every $1 \leq k \leq A$, a.s. for every $\tau$ large enough,

$$
\widehat{N}_{k}(\tau)>e^{w^{\prime} \tau} .
$$

and 
Lemma 7. There exists $v<w$ s.t. for every $1 \leq k<j \leq A$ a.s. for every $\tau$ large enough,

$$
p_{j} \widehat{N}_{k}(\tau)-p_{k} \widehat{N}_{j}(\tau)<e^{v \tau},
$$

where $p_{i}, i=1, \ldots, A$ are the components of the vector $\widehat{p}$.

The following corollary is an immediate consequence of Claim 2 Claim 1 and Lemma 6

Corollary 8. $w \leq 2$.

Proof of Lemma 7 Choose some $v$ strictly between $v^{\prime}$ and $w$ in a way that $w-v<0.25 \min \left(0.1, v-v^{\prime}, w / 10\right)$ and let $\delta=\min \left(0.1, v-v^{\prime}, w / 10\right)$. The vector

$$
b_{i}= \begin{cases}p_{j} & \text { if } i=k \\ -p_{k} & \text { if } i=j \\ 0 & \text { otherwise }\end{cases}
$$

satisfies $b^{\top} \hat{p}=0$, and therefore, using (26) and Markov's inequality,

$$
\mathbf{P}\left(p_{j} \widehat{N}_{k}(T)-p_{k} \widehat{N}_{j}(T)=b^{\top} \widehat{N}(T)>\frac{1}{3} e^{v T}\right) \leq 9 C e^{-2 \delta T} .
$$

Let $\left\{T_{i}\right\}_{i=1,2, \ldots}$ be such that $e^{2 \delta T_{i}}=i^{2}$. By Borel-Cantelli, almost surely there exists $i_{0}$ such that for all $i>i_{0}$,

$$
p_{j} \widehat{N}_{k}\left(T_{i}\right)-p_{k} \widehat{N}_{j}\left(T_{i}\right)<\frac{1}{2} e^{v T_{i}}
$$

Note that

$$
T_{i}=\frac{\log i}{\delta}
$$

and therefore

$$
T_{i+1}-T_{i}=\Theta\left(i^{-1}\right)
$$

We want to show that almost surely for all $T$ large enough,

$$
p_{j} \widehat{N}_{k}(T)-p_{k} \widehat{N}_{j}(T)<e^{v T}
$$

Section 3.3 tells us that $\mathbb{E}\left(\left\|\widehat{N}\left(T_{i}\right)\right\|\right)=O\left(\exp \left(w T_{i}\right)\right)$, and Lemma [ tells us that $\operatorname{var}\left(\left\|\widehat{N}\left(T_{i}\right)\right\|\right)=O\left(\exp \left(2 w T_{i}\right)\right)$. Therefore

$$
\mathbf{P}\left(\left\|\widehat{N}\left(T_{i}\right)\right\|>e^{(w+0.6 \delta) T_{i}}\right)<C_{l} e^{-1.2 \delta T_{i}}=C_{l} i^{-1.2}
$$

for some constant $C_{l}$, so that, if $m(i)$ is the number of vertices arriving between $T_{i}$ and $T_{i+1}$, then

$$
\begin{aligned}
& \mathbf{P}\left(m(i)>\frac{1}{2} e^{v T_{i}}\right) \\
& \quad \leq \mathbf{P}\left(\left\|\widehat{N}\left(T_{i}\right)\right\|>e^{(w+0.6 \delta) T_{i}}\right)+\mathbf{P}\left(m(i)>\frac{1}{2} e^{v T_{i}} \mid\left\|\widehat{N}\left(T_{i}\right)\right\| \leq e^{(w+0.6 \delta) T_{i}}\right) \\
& \quad \leq \mathbf{P}\left(\left\|\widehat{N}\left(T_{i}\right)\right\|>e^{(w+0.6 \delta) T_{i}}\right)+\mathbf{P}\left(m(i)>\frac{1}{2} e^{v T_{i}} \mid\left\|\widehat{N}\left(T_{i}\right)\right\|=e^{(w+0.6 \delta) T_{i}}\right) \\
& \quad \leq C_{l} i^{-1.2}+C_{s} e^{-(w+0.6 \delta) T_{i}}\left(T_{i+1}-T_{i}\right)^{-1}
\end{aligned}
$$


where the last inequality uses (14) in Claim 2 and the fact that

$$
\frac{1}{2} e^{v T_{i}}>2 e^{(w+0.6 \delta) T_{i}}\left(\exp \left(2\left(T_{i+1}-T_{i}\right)\right)-1\right)
$$

for $i$ large enough.

Clearly, the first part of the right side of (32) is a convergent sum. We need to show that so is the second part. Remember the choice $\delta \leq w / 10$. Then, using (30),

$$
\begin{aligned}
C_{s} e^{-(w+0.6 \delta) T_{i}}\left(T_{i+1}-T_{i}\right)^{-1} & =\Theta\left(i \cdot e^{-(w+0.6 \delta) T_{i}}\right) \\
=\Theta\left(e^{\delta T_{i}} \cdot e^{-(w+0.6 \delta) T_{i}}\right) & =\Theta\left(e^{-(w-0.4 \delta) T_{i}}\right) \\
=O\left(e^{-9 \delta T_{i}}\right) & =O\left(i^{-9}\right) .
\end{aligned}
$$

Using Borel-Cantelli, we conclude that almost surely,

$$
\sum_{k=1}^{A}\left|\widehat{N}_{k}(T)-\widehat{N}_{k}\left(T_{i}\right)\right|<\frac{1}{2} e^{v T_{i}}
$$

for all $k$ and all $i$ large enough and all $T$ between $T_{i}$ and $T_{i+1}$. Equation (31) follows from (33).

Proof of Lemma [6] By Lemma $\left[\operatorname{var}\left(\widehat{N}_{1}(\tau)\right)<C_{1} e^{2 w \tau}\right.$, while $\mathbb{E}\left(\widehat{N}_{1}(\tau)\right)>C_{2} e^{w \tau}$ by Section 3.3] Therefore there exists $\rho>0$ such that

$$
\mathbf{P}\left(\widehat{N}_{1}(\tau)>\rho e^{w \tau}\right)>\rho .
$$

Fix some large $T$, and let $\tau_{i}=i T$. For each vertex $v$ which is a fertile leaf at time $\tau_{i-1}$, let $\ell_{v}$ denote the number of descendants of $v$ (including $v$ itself) at time $\tau_{i}$ which are fertile leaves. The random variables $\left\{\ell_{v}\right\}$ are independent, their sum is $\widehat{N}_{1}\left(\tau_{i}\right)$, and the distribution of each of them is the same as the unconditional distribution of $\widehat{N}_{1}(T)$. Using this fact and (34), we get

$$
\mathbf{P}\left(\widehat{N}_{1}\left(\tau_{i}\right)>\frac{\rho^{2}}{2} e^{w T} \widehat{N}_{1}\left(\tau_{i-1}\right) \mid \widehat{N}_{1}\left(\tau_{i-1}\right)\right) \geq 1-e^{-\frac{1}{16} \widehat{N}_{1}\left(\tau_{i-1}\right)}
$$

via Chernoff's bound. From (35), we get that almost surely there exists a constant $C_{3}>0$ such that, for all $i$ large enough,

$$
\widehat{N}_{1}\left(\tau_{i}\right)>C_{3} \exp \left(i\left[w T+\log \left(\frac{\rho^{2}}{2}\right)\right]\right) .
$$

From Lemma 7 we may conclude that the same holds for $\widehat{N}_{A}\left(\tau_{i}\right)$, i.e. for any constant $C_{4}<C_{3}$,

$$
\widehat{N}_{A}\left(\tau_{i}\right)>C_{4} \exp \left(i\left[w T+\log \left(\frac{\rho^{2}}{2}\right)\right]\right) .
$$

$\widehat{N}_{A}(\tau)$ is monotone increasing, and therefore there exists $C_{5}>0$ such that

$$
\widehat{N}_{A}(\tau)>C_{5} \exp \left(\tau\left[w+\frac{1}{T} \log \left(\frac{\rho^{2}}{2}\right)\right]\right)
$$

for all $\tau$ large enough. Using Lemma $\mathbf{7}$ again, we conclude that there exists $C_{6}>0$ such that

$$
\widehat{N}_{k}(\tau)>C_{6} \exp \left(\tau\left[w+\frac{1}{T} \log \left(\frac{\rho^{2}}{2}\right)\right]\right)
$$


for all $k$ and large enough $\tau$. We get (27) by taking $T$ so large that

$$
w+\frac{1}{T} \log \left(\frac{\rho^{2}}{2}\right)>w^{\prime}
$$

Proposition 9. For every $k$ and $j$, almost surely

$$
\lim _{t \rightarrow \infty} \frac{\widehat{N}_{k}(\tau)}{\widehat{N}_{j}(\tau)}=\frac{p_{k}}{p_{j}}
$$

Proof. This follows immediately from Lemma 6 and Lemma 7

\subsection{Back to discrete time}

Proposition 10. For the discrete-time process, and $A>\max \left\{A_{1}, A_{2}\right\}$ there exists a vector $\hat{q}$ such that, for $k \leq A$, we have

$$
\lim _{t \rightarrow \infty} \frac{\widetilde{N}_{k}(t)}{t+1}=q_{k}
$$

Proof. The number of infertile vertices increases at step $t$ with probability

$$
\frac{\sum_{k=1}^{A_{1}-1} \widetilde{N}_{k}(t)}{\sum_{k=1}^{A} \widetilde{N}_{k}(t)}
$$

(their number cannot decrease). However, by (37), this expression tends to a limit, and therefore, using the law of large numbers,

$$
\lim _{t \rightarrow \infty} \frac{N_{0}(t)}{t+1}=q_{0}=\frac{\sum_{k=1}^{A_{1}-1} p_{k}}{\sum_{k=1}^{A} p_{k}}
$$

Using (37) once more, the proposition now follows for $k \geq 1$ with $q_{k}=\left(1-q_{0}\right) p_{k}$.

Note that the above proposition implies that $q_{k}$ and hence $p_{k}$ is independent of $A$ if $A>k$, since the left hand side of (38) does not depend on $A$ if $A>k$. So, in particular, $p_{1}$ does not depend on $A$.

\section{Power Law With a Cutoff}

In the previous section, we saw that for every $A>\max \left\{A_{1}, A_{2}\right\}$, the limiting proportions up to $A-1$ are $\lambda \hat{p}$ where $\hat{p}$ is the eigenvector corresponding to the highest eigenvalue $w$ of the $A$-by- $A$ matrix $M$ defined in Eqn. 11] Therefore, the components $p_{1}, p_{2}, \ldots, p_{A}$ of the vector $\hat{p}$ satisfy the equation:

$$
w p_{i}=-\min \left(i, A_{2}\right) p_{i}+\min \left(i-1, A_{2}\right) p_{i-1} \quad i \geq 2
$$

where the normalization is determined by $\sum_{i=1}^{A} p_{i}=1$. From (40) we get that for $i \leq A_{2}$,

$$
p_{i}=\left(\prod_{k=2}^{i} \frac{k-1}{k+w}\right) p_{1}
$$


and for $i>A_{2}$

$$
p_{i}=\left(\frac{A_{2}}{A_{2}+w}\right)^{i-A_{2}} p_{A_{2}}
$$

Clearly, (42) is exponentially decaying. There are many ways to see that (41) behaves like a power law with degree $1+w$. Indeed,

$$
\begin{aligned}
\frac{p_{i}}{p_{1}}=\left(\prod_{k=2}^{i} \frac{k-1}{k+w}\right) & =\exp \left(\sum_{k=2}^{i} \log \left(\frac{k-1}{k+w}\right)\right) \\
=\exp \left(\sum_{k=2}^{i}\left(\frac{-1-w}{k+w}\right)+O(1)\right) & =\exp \left((-1-w)\left(\sum_{k=2}^{i}(k+w)^{-1}\right)+O(1)\right) \\
=\exp \left((-1-w)\left(\sum_{k=2}^{i} k^{-1}\right)+O(1)\right) & =\exp \left((-1-w)\left(\sum_{k=2}^{i} \log \left(\frac{k+1}{k}\right)\right)+O(1)\right) \\
=\exp ((-1-w) \log (i / 2)+O(1)) & =O(1) i^{-1-w} .
\end{aligned}
$$

Note that the constants implicit in the $O(\cdot)$ symbols do not depend on $A_{1}, A_{2}$ or $i$, due the fact that $0<w \leq 2$. Equation (43) can be stated in the following way:

Proposition 11. There exist $0<c<C<\infty$ such that for every $A_{1}, A_{2}$ and $i \leq A_{2}$, if $w=w\left(A_{1}, A_{2}\right)$ is as in (40), then

$$
c i^{-1-w} \leq \frac{p_{i}}{p_{1}} \leq C i^{-1-w} .
$$

The vector $\left(q_{1}, q_{2}, \ldots, q_{A-1}\right)$ is a scalar multiple of the vector $\left(p_{1}, p_{2}, \ldots, p_{A-1}\right)$, so equations (5), ([6), and (7) in Theorem 2 (and the comment immediately following it) are consequences of equations (41), (42), and (44) derived above. It remains to prove the normalization conditions

$$
\sum_{i=0}^{\infty} q_{i}=1 \quad \text { and } \quad q_{0}=\sum_{i=1}^{\infty} q_{i} \min \left(i-1, A_{1}-1\right)
$$

stated in Theorem 2 These follow from the equations

$$
\sum_{i=0}^{\infty} N_{i}(t)=t+1 \quad \text { and } \quad N_{0}(t)=\sum_{i=1}^{\infty} N_{i}(t) \min \left(i-1, A_{1}-1\right) .
$$

The first of these simply says that there are $t+1$ vertices at time $t$; the second equation is proved by counting the number of infertile children of each fertile node.

\section{$5 \quad$ Monotonicity Properties of $w$}

In this section we will prove that the exponent $1+w$ of the power law in Proposition [1] is monotonically decreasing in $A_{1}$ and monotonically increasing in $A_{2}$. For this purpose, it will be useful to define a family of matrices, parameterized by two vectors $\mathbf{y}, \mathbf{z} \in \mathbb{R}^{n}$, which generalizes the matrix $M$ appearing in (11), whose top eigenvalue is $w$. 
Given vectors $\mathbf{y}=\left(y_{1}, y_{2}, \ldots, y_{n}\right), \mathbf{z}=\left(z_{1}, z_{2}, \ldots, z_{n}\right) \in \mathbb{R}^{n}$, let $M(\mathbf{y}, \mathbf{z})$ denote the $n$-by- $n$ matrix whose (ij)-th entry is:

$$
M_{i, j}(\mathbf{y}, \mathbf{z})= \begin{cases}z_{1}-y_{1} & \text { if } 1=i=j \\ -y_{j} & \text { if } 2 \leq i=j \leq n \\ y_{j} & \text { if } 2 \leq i=j+1 \leq n \\ z_{j} & \text { if } i=1 \text { and } j \geq 2 \\ 0 & \text { otherwise. }\end{cases}
$$

Thus, for instance, the matrix $M$ defined in (11) is $M(\mathbf{y}, \mathbf{z})$, where $n=A$ and

$$
\begin{aligned}
& y_{j}= \begin{cases}\min \left(j, A_{2}\right) & \text { if } 1 \leq j<A \\
0 & \text { if } j=A\end{cases} \\
& z_{j}= \begin{cases}0 & \text { if } 1 \leq j<A_{1} \\
\min \left(j, A_{2}\right) & \text { if } A_{1} \leq j \leq A .\end{cases}
\end{aligned}
$$

For the remainder of this section, we will assume:

$$
\begin{aligned}
& \text { - } y_{i}>0 \text { for } 1 \leq i<n, \\
& \text { - } z_{i} \geq 0 \text { for } 1 \leq i<n, \\
& \text { - } y_{n}=0, z_{n}>0 .
\end{aligned}
$$

All of these criteria will be satisfied by the matrices $M(\mathbf{y}, \mathbf{z})$ which arise in proving the desired monotonicity claim. It follows from (45), (46), and (47) that if we add a suitably large scalar multiple of the identity matrix to $M(\mathbf{y}, \mathbf{z})$, we obtain an irreducible matrix $M(\mathbf{y}, \mathbf{z})+B I$ with non-negative entries. The Perron-Frobenius Theorem guarantees that $M(\mathbf{y}, \mathbf{z})+B I$ has a positive real eigenvalue $R$ of multiplicity 1 , such that all other complex eigenvalues have modulus $\leq R$; consequently $M(\mathbf{y}, \mathbf{z})$ has a real eigenvalue $w=R-B$, of multiplicity 1 , such that the real part of every other eigenvalue is strictly less than $w$.

We will study how $w$ varies under perturbations of the parameters $\mathbf{y}, \mathbf{z}$. Let $P(\lambda, \mathbf{y}, \mathbf{z})$ be the characteristic polynomial of $M(\mathbf{y}, \mathbf{z})$, i.e.

$$
P(\lambda, \mathbf{y}, \mathbf{z})=\operatorname{det}(\lambda I-M(\mathbf{y}, \mathbf{z})) .
$$

This is a polynomial of degree $n$ in $\lambda$ (with coefficients depending smoothly on $\mathbf{y}, \mathbf{z}$ ), whose largest real root $w(\mathbf{y}, \mathbf{z})$ exists and has multiplicity 1 , provided $(\mathbf{y}, \mathbf{z})$ belongs to the region $V \subset \mathbb{R}^{n} \times \mathbb{R}^{n}$ determined by (45), (46), and (47). It follows from the implicit function theorem that $w(\mathbf{y}, \mathbf{z})$ is a smooth function of $(\mathbf{y}, \mathbf{z})$ in $V$, satisfying:

$$
\left.\left(\frac{\partial P}{\partial y_{i}}+\frac{\partial w}{\partial y_{i}} \cdot \frac{\partial P}{\partial \lambda}\right)\right|_{(w, \mathbf{y}, \mathbf{z})}=0 ;\left.\quad\left(\frac{\partial P}{\partial z_{i}}+\frac{\partial w}{\partial z_{i}} \cdot \frac{\partial P}{\partial \lambda}\right)\right|_{(w, \mathbf{y}, \mathbf{z})}=0
$$

If $\mathbf{x}$ is any vector in $\mathbb{R}^{n} \times \mathbb{R}^{n}$, and $\partial_{\mathbf{x}}$ is the corresponding directional derivative operator, we have from (48):

$$
\partial_{\mathbf{x}} w(\mathbf{y}, \mathbf{z})=-\frac{\partial_{\mathbf{x}} P(w, \mathbf{y}, \mathbf{z})}{\left.(\partial P / \partial \lambda)\right|_{(w, \mathbf{y}, \mathbf{z})}}
$$

We know that $\left.(\partial P / \partial \lambda)\right|_{(w, \mathbf{y}, \mathbf{z})}>0$ because $P$ is a polynomial with positive leading coefficient, $w$ is its largest real root, and $w$ has multiplicity 1 . Thus we have established: 
Claim 3. For any vector $\mathbf{x} \in \mathbb{R}^{n} \times \mathbb{R}^{n}$, and any $(\mathbf{y}, \mathbf{z}) \in V$, put $w=w(\mathbf{y}, \mathbf{z})$. Then the directional derivatives $\partial_{\mathbf{x}} w(\mathbf{y}, \mathbf{z})$ and $\partial_{\mathbf{x}} P(w, \mathbf{y}, \mathbf{z})$ have opposite signs.

This allows monotonicity properties of $w$ to be deduced from calculations involving directional derivatives of $P$. Given the definition of $M(\mathbf{y}, \mathbf{z})$, it is straightforward to compute that

$$
P(\lambda, \mathbf{y}, \mathbf{z})=\operatorname{det}(\lambda I-M(\mathbf{y}, \mathbf{z}))=P_{1}(\lambda, y, z)-\sum_{j=2}^{n} P_{j}(\lambda, \mathbf{y}, \mathbf{z}),
$$

where

$$
\begin{aligned}
& P_{1}(\lambda, y, z)=\left(\lambda+y_{1}-z_{1}\right) \prod_{i=2}^{n}\left(\lambda+y_{i}\right) \\
& P_{j}(\lambda, \mathbf{y}, \mathbf{z})=\left(\prod_{i=1}^{j-1} y_{i}\right) z_{j}\left(\prod_{i=j+1}^{n}\left(\lambda+y_{j}\right)\right) .
\end{aligned}
$$

As an easy consequence of this formula, $w$ is strictly positive.

Lemma 12. $w$ is strictly positive.

Proof. From (501)-(52) and the fact that $y_{n}=0$, we have $P(0, \mathbf{y}, \mathbf{z})=-P_{n}(0, \mathbf{y}, \mathbf{z})=-\left(\prod_{i=1}^{n-1} y_{i}\right) z_{n}$, and this is strictly negative by (45) and (47). For sufficiently large positive $\lambda$, we know that $P(\lambda, \mathbf{y}, \mathbf{z})>0$ because $P$ is a polynomial whose leading coefficient in $\lambda$ is positive. By the intermediate value theorem, $P(\lambda, \mathbf{y}, \mathbf{z})$ has a strictly positive real root.

The following three lemmas encapsulate the requisite directional derivative estimates for $P$.

Lemma 13. $\left.\left(\partial P / \partial z_{k}\right)\right|_{(w, \mathbf{y}, \mathbf{z})}<0$ for $(\mathbf{y}, \mathbf{z}) \in V$.

Proof. For $k>1$,

$$
\partial P / \partial z_{k}=-\partial P_{k} / \partial z_{k}=-\left(\prod_{i=1}^{k-1} y_{i}\right)\left(\prod_{i=k+1}^{n}\left(w+y_{i}\right)\right)<0 .
$$

For $k=1$,

$$
\partial P / \partial z_{1}=\partial P_{1} / \partial z_{1}=-\prod_{i=2}^{n}\left(w+y_{i}\right)<0 .
$$

Corollary 14. $w$ is monotonically decreasing in $A_{1}$.

Proof. Increasing $A_{1}$ from $k$ to $k+1$ has no effect on $\mathbf{y}$, and its only effect on $\mathbf{z}$ is to decrease $z_{k}$ from $\min \left(k, A_{2}\right)$ to 0 . As we move in the $-z_{k}$ direction, the directional derivative of $P$ is positive, so the directional derivative of $w$ is negative by Claim 3 Thus $w$ decreases as we increase $A_{1}$ from $k$ to $k+1$.

Lemma 15. For $1<k<n,\left.\left(\partial P / \partial y_{k}\right)\right|_{(w, \mathbf{y}, \mathbf{z})}<0$ if $(\mathbf{y}, \mathbf{z}) \in V$ and $z_{k}=0$. 
Proof.

$$
\begin{aligned}
\frac{\partial P}{\partial y_{k}} & =\frac{\partial P_{1}}{\partial y_{k}}-\sum_{j=2}^{n} \frac{\partial P_{j}}{\partial y_{k}} \\
& =\frac{1}{w+y_{k}} P_{1}-\frac{1}{w+y_{k}} \sum_{j=2}^{k-1} P_{j}-\frac{1}{y_{k}} \sum_{j=k+1}^{n} P_{j} \\
& <\frac{1}{w+y_{k}} P_{1}-\frac{1}{w+y_{k}} \sum_{j=2}^{k-1} P_{j}-\frac{1}{w+y_{k}} \sum_{j=k+1}^{n} P_{j} \\
& =\frac{P(w, \mathbf{y}, \mathbf{z})}{w+y_{k}} \\
& =0
\end{aligned}
$$

Lemma 16. For $k>1,\left.\left(\partial P / \partial y_{k}+\partial P / \partial z_{k}\right)\right|_{(w, \mathbf{y}, \mathbf{z})}<0$ if $(\mathbf{y}, \mathbf{z}) \in V$ and $y_{k}=z_{k}$.

Proof.

$$
\begin{aligned}
\frac{\partial P}{\partial y_{k}}+\frac{\partial P}{\partial z_{k}} & =\frac{\partial P_{1}}{\partial y_{k}}-\sum_{j=2}^{n} \frac{\partial P_{j}}{\partial y_{k}}-\frac{\partial P_{k}}{\partial z_{k}} \\
& =\frac{1}{w+y_{k}} P_{1}-\frac{1}{w+y_{k}} \sum_{j=2}^{k-1} P_{j}-\frac{1}{y_{k}} \sum_{j=k+1}^{n} P_{j}-\frac{1}{z_{k}} P_{k} \\
& <\frac{1}{w+y_{k}} P_{1}-\frac{1}{w+y_{k}} \sum_{j=2}^{k-1} P_{j}-\frac{1}{w+y_{k}} \sum_{j=k+1}^{n} P_{j}-\frac{1}{w+y_{k}} P_{k} \\
& =\frac{P(w, \mathbf{y}, \mathbf{z})}{w+y_{k}} \\
& =0
\end{aligned}
$$

Corollary 17. $w$ is monotonically increasing in $A_{2}$.

Proof. If we change $A_{2}$ from $k$ to $k+1$, this changes $\mathbf{y}$ into a new vector $\mathbf{y}^{\prime}$ satisfying

$$
y_{j}^{\prime}-y_{j}= \begin{cases}1 & \text { if } k<j<n \\ 0 & \text { otherwise }\end{cases}
$$

It changes $\mathbf{z}$ into a new vector $\mathbf{z}^{\prime}$ satisfying

$$
z_{j}^{\prime}-z_{j}= \begin{cases}1 & \text { if } \max \left(A_{1}, k+1\right) \leq j \leq n \\ 0 & \text { otherwise }\end{cases}
$$

Letting $\mathbf{e}_{j}^{(y)}$ denote a unit vector in the $+y_{j}$ direction, and $\mathbf{e}_{j}^{(z)}$ a unit vector in the $+z_{j}$ direction, the direction of change is expressed by the vector

$$
\mathbf{x}=\left(\mathbf{y}^{\prime}, \mathbf{z}^{\prime}\right)-(\mathbf{y}, \mathbf{z})=\left[\sum_{k+1 \leq j<A_{1}} \mathbf{e}_{j}^{(y)}\right]+\left[\sum_{\max \left(k+1, A_{1}\right) \leq j<n}\left(\mathbf{e}_{j}^{(y)}+\mathbf{e}_{j}^{(z)}\right)\right]+\mathbf{e}_{n}^{(z)},
$$


and $\partial_{\mathbf{x}} P$ is negative, by the preceding three lemmas. By Claim 3 this means $w$ increases monotonically as we move along this path.

\section{Acknowledgment}

We wish to thank the referee for many good suggestions that helped us improve the paper.

\section{References}

[1] W. Aiello, F. Chung, and L. Lu. Random evolution of massive graphs. In Handbook of Massive Data Sets, pages 97-122. Kluwer, 2002.

[2] R. Albert and A.-L. Barabási. Statistical mechanics of complex networks. Rev. Mod. Phys., 74:47-97, 2002.

[3] D. J. Aldous. A stochastic complex network model. Electron. Res. Announc. Amer. Math. Soc., 9:152161, 2003.

[4] A.-L. Barabási and R. Albert. Emergence of scaling in random networks. Science, 286:509-512, 1999.

[5] N. Berger, B. Bollobás, C. Borgs, J. T. Chayes, and O. Riordan. Degree distribution of the FKP network model. In International Colloquium on Automata, Languages and Programming, 2003.

[6] N. Berger, C. Borgs, J. T. Chayes, R. M. D’Souza, and R. D. Kleinberg. Competition-Induced Preferential Attachment. In International Colloquium on Automata, Languages and Programming, 2004.

[7] B. Bollobás, C. Borgs, J. T. Chayes, and O. Riordan. Directed scale-free graphs. In Proceedings of the 14th ACM-SIAM Symposium on Discrete Algorithms, pages 132-139, 2003.

[8] B. Bollobás and O. Riordan. Mathematical results on scale-free random graphs. In Handbook of Graphs and Networks, Berlin, 2002. Wiley-VCH.

[9] B. Bollobás, O. Riordan, J. Spencer, and G. E. Tusnady. The degree sequence of a scale-free random graph process. Random Structures and Algorithms, 18:279-290, 2001.

[10] J. M. Carlson and J. Doyle. Highly optimized tolerance: a mechanism for power laws in designed systems. Phys. Rev. E, 60:1412, 1999.

[11] C. Cooper and A. M. Frieze. A general model of web graphs. In Proceedings of 9th European Symposium on Algorithms, pages 500-511, 2001.

[12] S. N. Dorogovtsev and J. F. F. Mendes. Evolution of networks. Adv. Phys., 51:1079, 2002.

[13] F. Eggenberger and G. Pólya. Über die statistik verketteter. Vorgänge. Zeitschrift Agnew. Math. Mech., 3:279-289, 1923. 
[14] A. Fabrikant, E. Koutsoupias, and C.H. Papadimitriou. Heuristically optimized trade-offs: a new paradigm for power laws in the internet. In International Colloquium on Automata, Languages and Programming, pages 110-122, 2002.

[15] M. Faloutsos, P. Faloutsos, and C. Faloutsos. On the power-law relationships of the Internet topology. Comput. Commun. Rev., 29:251, 1999.

[16] R. Govindan and H. Tangmunarunkit. Heuristics for Internet map discovery. In Proceedings of INFOCOM, pages $1371-1380,2000$.

[17] C. Kenyon and N. Schabanel. Personal communication.

[18] R. Kumar, P. Raghavan, S. Rajagopalan, D. Sivakumar, A. Tomkins, and E. Upfal. Stochastic models for the web graph. In Proc. 41st IEEE Symp. on Foundations of Computer Science, pages 57-65, 2000.

[19] M. E. J. Newman. The structure and function of complex networks. SIAM Review, 45:167-256, 2003.

[20] D. J. de S. Price. A general theory of bibliometric and other cumulative advantage processes. J. Amer. Soc. Inform. Sci., 27:292-306, 1976.

[21] H. A. Simon. On a class of skew distribution functions. Biometrika, 42(3/4):425-440, 1955.

[22] G. U. Yule. A mathematical theory of evolution, based on the conclusions of Dr. J. C. Willis. Philos. Trans. Roy. Soc. London, Ser. B 213:21-87, 1924.

[23] G. K. Zipf. Human Behavior and the Principle of Least Effort. Addison-Wesley, Cambridge,MA, 1949. 